\title{
Demand and Schedule Operation Mode for Personal Rapid Transit
}

\author{
Jong-Gyu Hwang *, Baek-Hyun Kim **, Seok-Won Kang***, and Rak Gyo Jeong***
}

\begin{abstract}
The PRT (Personal Rapid Transit) system is a new transportation system in accordance with the requirements of the passengers. Although this PRT system was proposed a long time ago, it did not hold the limelight due to the several limitations in technology. However, the research and development has been active again recently thanks to the development in technology and the review on its applicability as the means of transportation linking to the urban transit system. Although most of these recent studies in relation to the PRT are focused on the hardware such as PRT vehicles or track structures, etc., the operational aspect of it is more important than that of any other means of public transportation. Therefore, among studies on operational aspect of this PRT system, this paper shows the result of study on technology in relation to the movement of vehicle to the station where it was called by the passenger through interface of the wayside control center with the vehicle control system in case where the passenger calls a vehicle at the station.
\end{abstract}

Keywords: Personal Rapid Transit, Schedule Mode, Demand Mode

\section{Introduction}

As a new means of public transportation will be operated along the route and the schedule according to the demand of passengers, PRT system is a track car with unmanned automatic operation is possible[1][2]. Unlike existing general railways, PRT system is the new urban transit system which is operated non-stop to the destination of passengers on an on-demand basis by being operated in the method of off-line station based on operation lines in the form of complex networks. The biggest difference between the PRT system and existing railway system is the fact that it is the system where the vehicle is operated along the lines consisted of off-line stations and networks. Off-line station means that it can go non-stop to the destination wanted by it, and it means that it can go anywhere wanted by the passengers along the operation line consisted of networks. That is, it means the public transportation that can go nonstop to the destination wanted by passengers directly [3]-[5].

Fig. 1 shows the conceptual diagram for application of this PRT system, and it shows a PRT system as the means of personal transfer linked to the urban transit station. That is, it is the personal means of transportation which can be operated non-stop to the corresponding destination station if a random destination is wanted by the passenger on the PRT

\footnotetext{
* Korea Railroad Research Institute, Korea(jghwang@krri.re.kr)

** Korea Railroad Research Institute, Korea(\{bhkim, swkang, rgjeong@krri.re.kr)

Received 08 April 2013; Accepted 21 August 2013
}

line. This PRT vehicle is a non-stop service on-demand transit system that can transfer to the passenger destination station without transfer at intermediate stations. This PRT system has been pilot applying at some overseas, but it's hard to see that those are yet to have an on-demand attribute of PRT system on-demand.

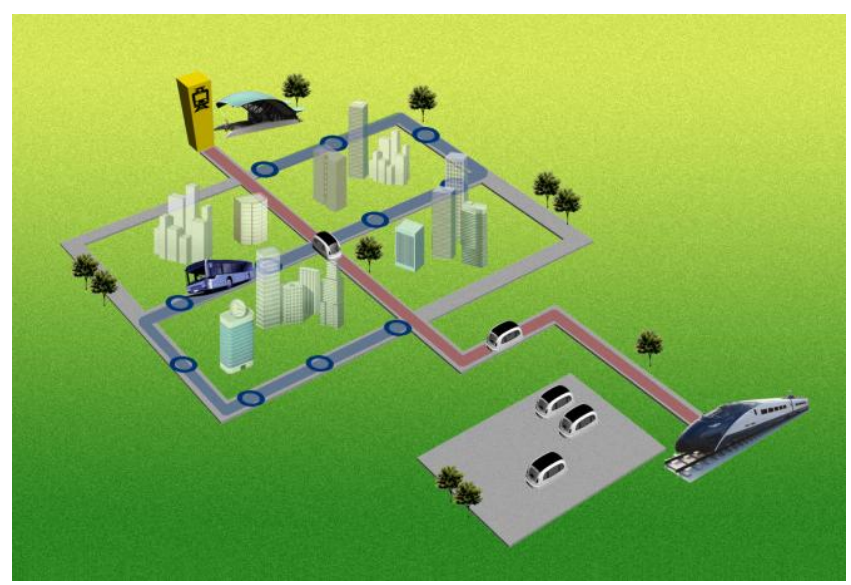

Fig. 1. Concept of optimal routing for PRT system

The related studies and development on PRT system are currently concentrated to the PRT vehicle technology. But the studies on on-demand characteristics are more important than vehicle development in PRT system research. It have been studied the development of Korea PRT systems from a few years ago in Korea. As part of these studies, this paper represents the operation scenarios on ondemand characteristics of the PRT system. In this paper, the 
operation scenario was studied on how the empty vehicle arrived to the called station when passenger calls the PRT vehicle at the station. The PRT car can be routing directly through optimal path for destination station to meet ondemand request of passengers [5].

\section{The PRT Operation Systems}

The on-demand functions are implemented by the PRT operation system. The PRT operation system shows in Fig. 2. Like this figure, this operation system to implement functions of PRT system can be divided into the Central Control System (CCS), Station Control System (SCS) and Wayside Communication System (WCS) basically.

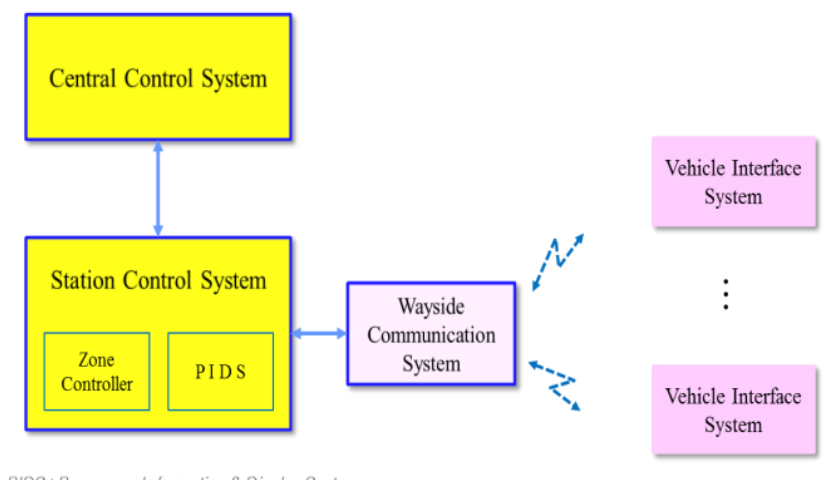

Fig. 2. System configuration of PRT system

For interfaces among each subsystem, and to deliver the control command from central control system to vehicle control system, CCS, SCS and WCS are connected by wire communication networks.

Among of them, the station control system consists of the zone controller and PIDS (Passenger Information and Display System). The zone controller will be located only in the base station. That is, the regular station, which is majority, only has the PIDS equipment, but the zone controller is installed at the base station. Zone controller performs control and supervision functions of vehicle through CCS and the interface to PRT vehicle existing within the corresponding area. PIDS is a device handling the function of passengers' call for vehicle and performing the function to guide and display passenger information.

Fig. 3 shows the configuration of PRT operation system which is installed and operated differently by each station. That is, unlike stations for other urban transit system, since stations for PRT have short distances between stations or they are operated in the form of bus stop, they are operated by dividing them as the base station and general station. In case of the base station, all of the control systems including zone controllers are installed, and in case of the temporary station, basic devices to provide passenger information will be operated only.

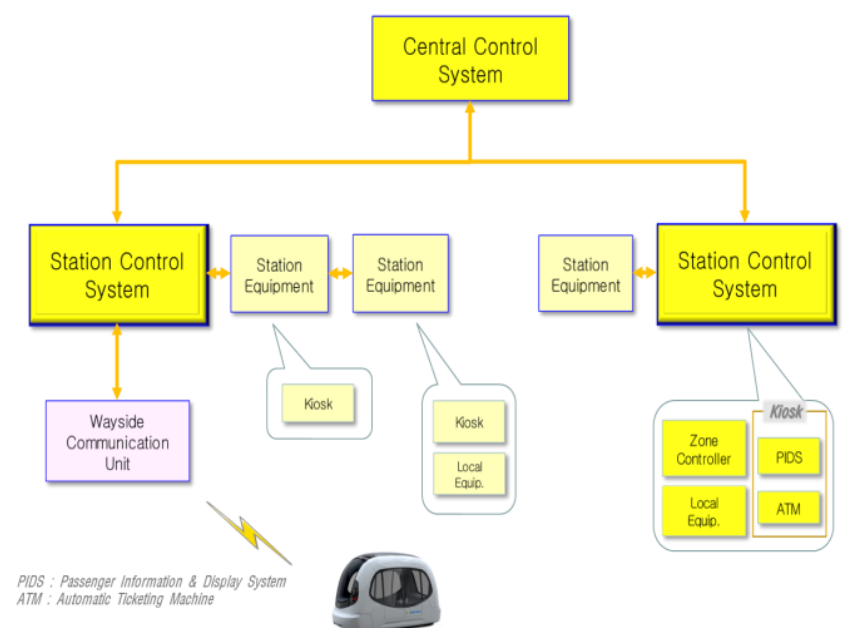

Fig. 3. Operation system where the base station and temporary station are distinguished

\section{Operation Modes on PRT System}

\subsection{Demand Mode}

Fig. 4 shows the conceptual data flow when a passenger calls the vehicle at the station to go his/her destination. The information is transmitted to the central controller for calling a car through PIDS at a station. The central control system checks the closest empty vehicle and then move to the vehicle to the called station for passenger.

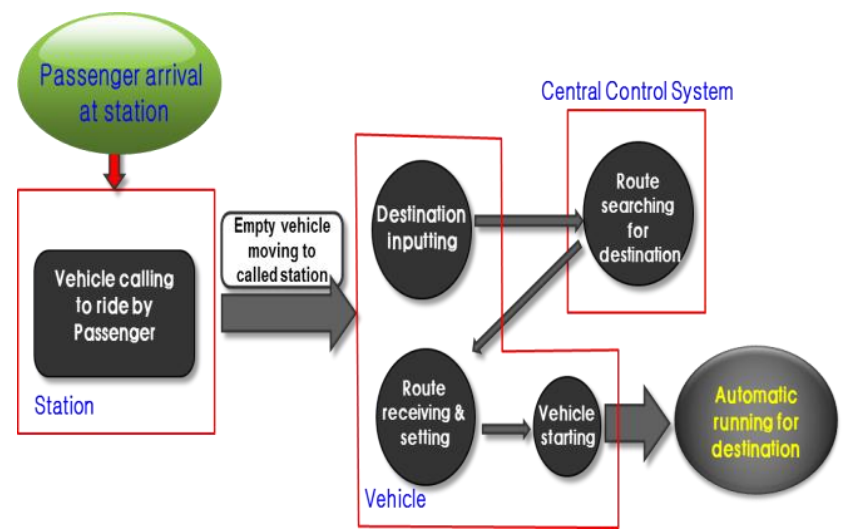

Fig. 4. Conceptual data flow for care calling

Fig. 5 shows a mechanism which operates for passenger car calling at the station through the data flow between subsystem of operation system. The data flow on car calling illustrates number (1) in Fig.5, and (2) means the running state of empty car for called station. The number (3) flow shows the inputting of destination station by passenger on vehicle and optimal route searching data by central control 
system to car. The CCS has a role to check the available car, to transfer the optimal route for empty car, and make moving command of empty car for called station in response to the car calling of passenger. When passengers on board the PRT car inputs a destination station, the CCS plays a role to find the optimal route with non-stop to the destination and be transmitted to the car control system.

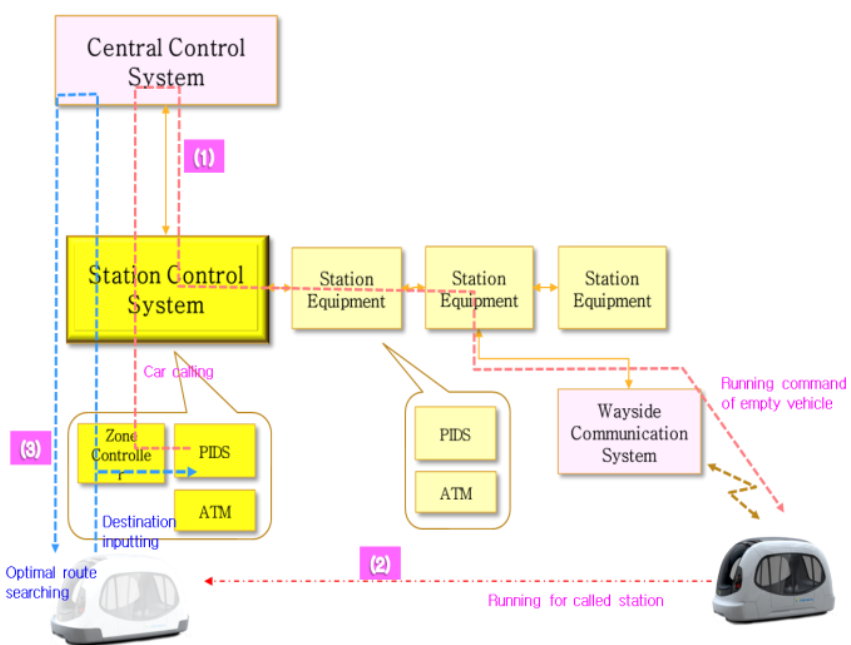

Fig. 5. Interface between Subsystem when Car Calling

The inter faces with the central control system and other sub-systems in the PRT system are a very important part at PRT system operating, as shown in Fig. 5. The analysis and definition of interface is more important because of the aim of fully automatic operation for PRT system.

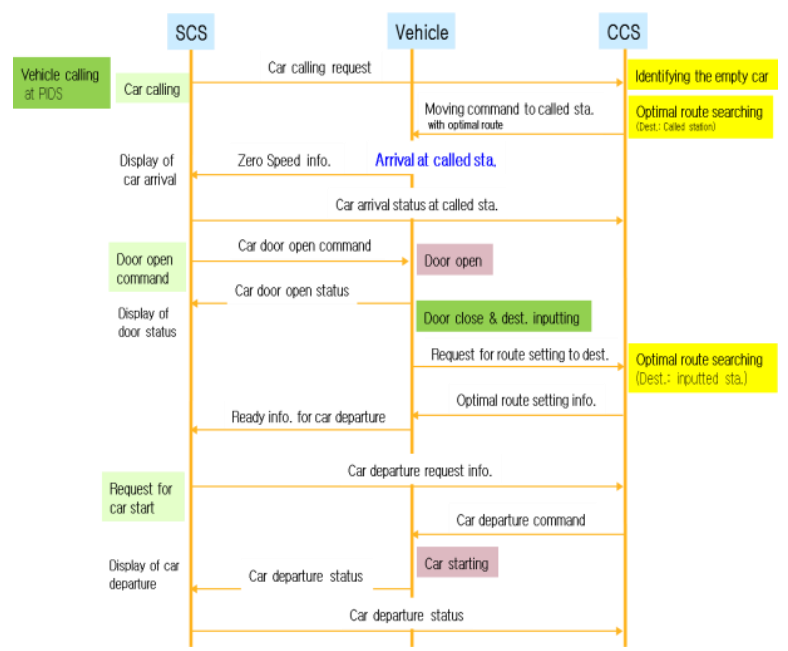

Fig. 6. Interface between subsystem when car calling

When the PRT car arrived at the station waiting passenger, the passenger will board the vehicle and input his/her destination to vehicle display panel. And then the vehicle request his running route to central control system via station control system. The central control system will explore the optimal route to final destination and then transmitted the results to vehicle interface system via station control system. The vehicle will start for destination through received route. In this paper, the interface protocol between each system, required to operate the PRT system until the departure from arrival of the passenger at a station, is analyzed and designed. Fig. 6 shows the flow of data based protocol designed like above description, and this was designed based on standard protocols for Korean railway.

\subsection{Schedule Mode}

In case of the schedule mode, the vehicle slightly larger than that for the demand mode explained previously and slightly smaller than the bus will be used, and unlike the previous mode, the vehicle will be operated on the determined route. In case of this schedule mode, it can be applicable to the certain day of the week or time zone such as the commuting time zone, etc., if a PRT system is applied which is seen as the means of linked transfer of the station building for urban transit.

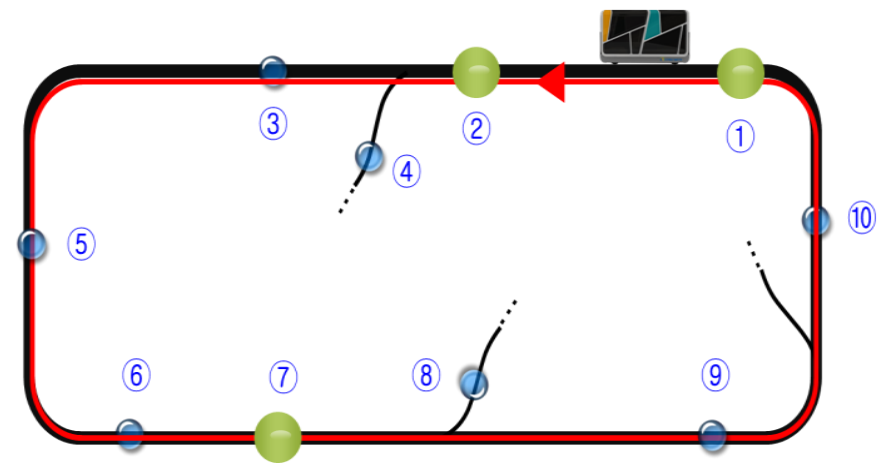

Fig. 7. Outline of the schedule operation mode

Fig. 7 shows the schedule operation mode. Here, the operation route of vehicle is set to be operated on the circular route in the figure. In the figure, in case where the passenger calls a vehicle at the wayside (1) and (7) stations like a demand mode, and if the passengers boarded on the vehicle request to get off at (2) and (9) stations, the vehicle will be operated on the route of (1) $\Rightarrow$ (2) $\Rightarrow$ (7) $\Rightarrow$ (1) by reflecting these two conditions, and it will be stopped at these stations. And it will bypass other remaining stations such as (3), (5), (6), (9) and (10) stations without stoppage.

Fig. 8 is the figure which compares this schedule mode of PRT with the demand mode. In the figure, A PRT vehicle is the vehicle being operated in the demand mode explained previously, and B PRT means the vehicle being operated in the schedule mode. If the destination of A PRT is the number (7) station, it will be operated on the shortest route 
of (1) $\rightarrow$ (9) $\rightarrow$ (6) $\rightarrow$ (7) route, and (9) \& (6) stations in the middle will be bypassed without stoppage. However, in case of $\mathrm{B}$ vehicle whose destination station is the (8) station, it will be driven on (2) $\rightarrow$ (4) $\rightarrow$ (6) $\rightarrow$ (8) routes determined previously. In this case, if there is any request for getting off at the station on the determined route or from the vehicle being operated since the demand function was added in the schedule mode, it will be stopped at the corresponding station. And if there is no request from the passenger at the wayside station or from the inside of vehicle, the corresponding station will be bypassed like that for demand mode.

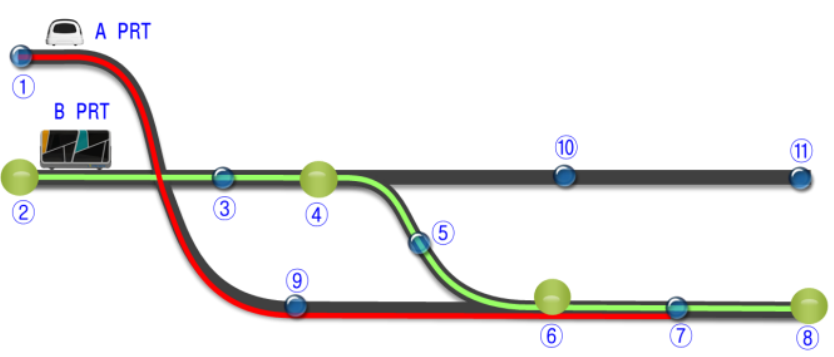

Fig. 8. Comparison of schedule mode with demand mode

\section{Testing at PRT System Test Line}

Currently at the KRRI, tests on two kinds of operation modes explained in this paper are under progress by building a PRT test line with a fixed length. In case of the current test line, we have a plan to expand it continuously. Therefore, in case of the operation system under development, the design and development are under progress accordingly taking into consideration of its expandability not of the fixed operation line. Fig. 9 shows a part of route editing algorithm at the operation system that has this expandability, which expresses the system algorithm that has functions such as the operational direction of line, route setting, and addition and deletion of station, etc.

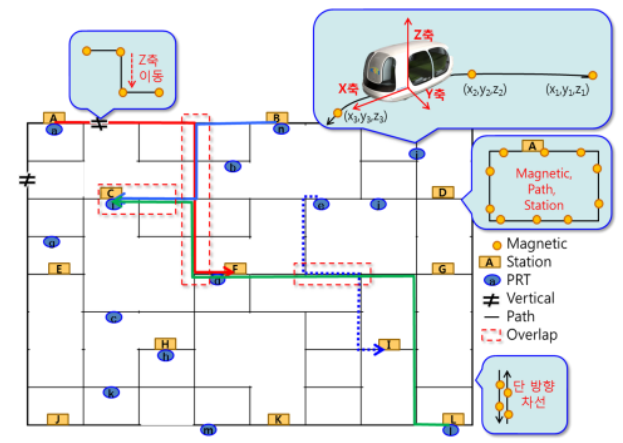

Fig. 9. Operation line editing module with expandability
Passengers wanted to go to a station can be call the empty PRT vehicle by this PIDS equipment installed in the station. Currently, the basic functional test is being carried out in the test line with installed facilities like Fig. 5 and 6. Also, the verification test is running in this test line on various operational scenarios of PRT system, including those described in this paper.

And also the test line for PRT system has been built like Fig. 10 and has being tested at the test line. The subsystem of PRT operation was installed to the test line, as shown in Fig. 10 such as CCS, SCS and Vehicle wireless I/F unit. The wireless WIFI network was built to cover the entire test line for the interface of the vehicle and on the ground equipment, and also the wireless interface was built in vehicle. Fig. 11 shows the equipment of PIDS installed in the station to offer the information of vehicle operating status and to call the vehicle by wanted to ride a the PRT vehicle.

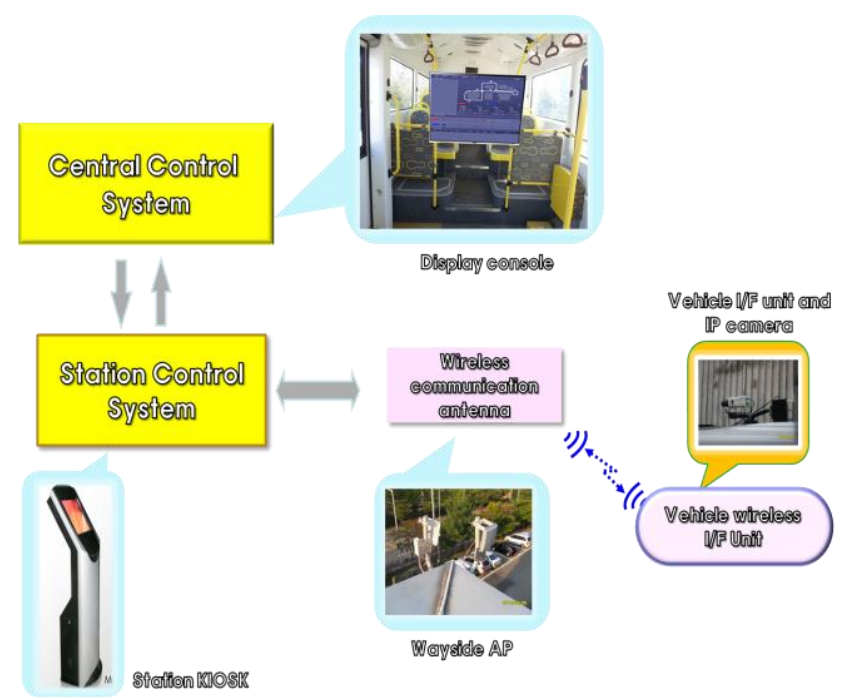

Fig. 10 System Configuration for Testing in PRT Test Line

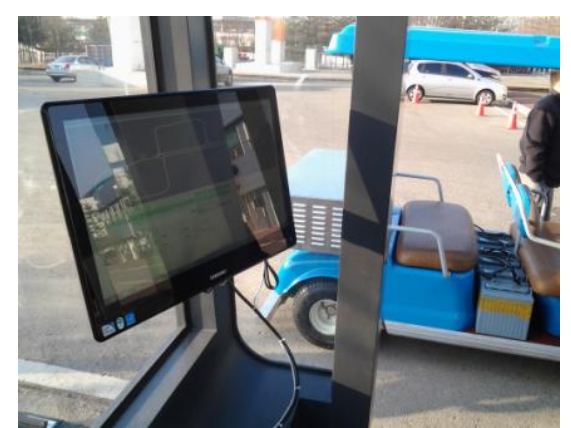

Fig. 11. The installed PIDS in the station

Passengers wanted to go to a station can be call the empty PRT vehicle by this PIDS equipment installed in the station. Currently, the basic functional test is being carried out in the test line with installed facilities like Figs. 10 and 
11. Also, the verification test is running in this test line on various operational scenarios of PRT system, including those described in this paper.

Fig. 12 shows the operational screen of PIDS installed in the station, and yellow color means the route on which the vehicle will be driven. The information on operating speed of vehicle final destination station, distance to move and remaining distance to the destination, etc. is displayed on the screen of PIDS since functions for operation modes are under test in the test line currently, and in case where this system is commercialized in the future, this information is planned to be monitored at the control room only.

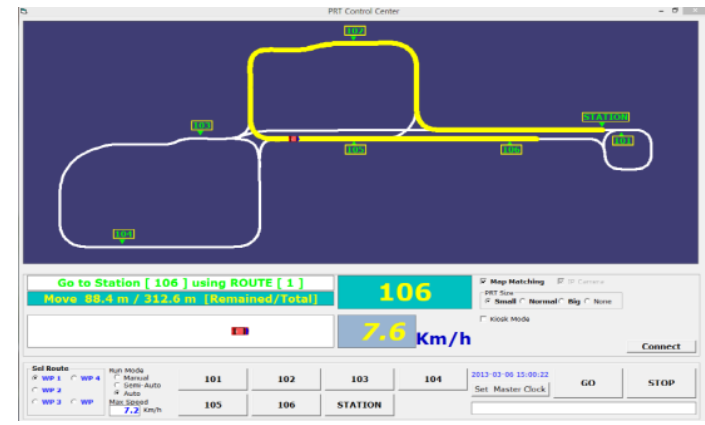

Fig. 12. Screen of PIDS in the station

\section{References}

[1] http://transportation.wvu.edu/prt/

[2] J. E. Anderson, "The Future of High-Capacity PRT," Proceedings of Advanced Automated Transit Systems Conference, Bologna, Italy, November 7-8, 2005.

[3] S. Shladover, Automated vehicle control developments in the PATH program" IEEE Transactions on Vehicular Technology, Vol. 40, Issue 1, Feb. 1991.

[4] Kim H., Oh J-S, R. Jayakrishnan, "Application of activity chaining model incorporating a time use problem to network demand analysis", Transportation Research Record No. 1977, 2006.

[5] KRRI Research Report, "Development of Technolgies for On-demand Circulator Transit System(PRT)", Korea Railroad Research Institute, 2012.

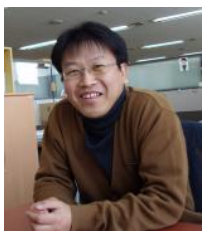

Jong-Gyu Hwang received the B.S and M.S. degrees in Electrical Engineering from Konkuk University, Korea in 1994 and 1996, respectively. He has been working towards his Ph.D. in the Division of Electrical and Computer Engineering, Hanyang University since the year 2000. As of 1995, he has been a Principal Researcher with the Korea Railroad Research Institute. He was a visiting scholar at Virginia Commonwealth Univ. from 2011 to 2012. His research interests are in the areas of railway signaling system, computer network technology, PRT (Personal Rapid Transit) system, software testing of embedded system.

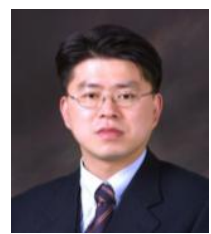

Back-Hyun Kim received the B.S., M.S., and Ph.D. degrees, all in Electronic Engineering, from Inha University, Incheon, South Korea, in 1994, 1996, and 2003, under the Korean Air Line Scholarship Grants, respectively. He joined Train Control and Communication Research Department at KRRI (Korea Railroad Research Institute), Uiwang, South Korea, as a Senior Researcher in 2003. Currently, he is working for On-demand Transit Research Team in the New Transportation Systems Research Center at KRRI. His research interests include multiple access communication systems, railway communications, train control, and PRT (Personal Rapid Transit) network operation.

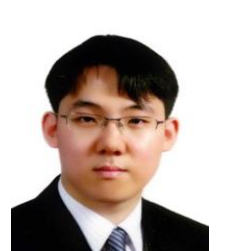

Seok-Won Kang received the B.S. degree in Mechanical Engineering with a minor in Electrical and Computer Engineering from Hanyang University, Seoul, South Korea, in 2005, the M.S. degree in Mechanical Aerospace \& Systems Engineering from KAIST (Korea Advanced Institute of Science and Technology), Daejeon, South Korea, in 2007, and the Ph.D. degree in Mechanical Engineering from Texas A\&M University, College Station, TX, USA, in 2012. Currently, he is a Senior Researcher working for On-demand Transit Research Team in the New Transportation Systems Research Center at KRRI (Korea Railroad Research Institute), Uiwang, South Korea, since 2012. From 2007 to 2008, he was a Research Engineer in R\&D division at Renault Samsung Motors, Yongin, South Korea. His research interests include Micro/Nano-scale thermo-fluidics, design of MEMS sensor, Bio-MEMS (Lap-On-Chip), $\mu$ TAS and numerical methods in engineering.

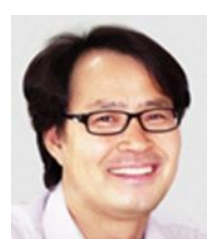

Rag-Gyo Jeong received the B.S., M.S., and Ph.D. degrees, all in Electrical Engineering, from Inha University, Incheon, South Korea, in 1991, 1999, and 2005, respectively. He joined KRRI (Korea Railroad Research Institute), Uiwang, South Korea, as a Senior Researcher in 1995. Currently, he is a Principal Researcher and team leader of On-demand Transit Research Team in the New Transportation Systems Research Center at KRRI. From 1990 to 1994, he was a Staff Engineer at Hanjin Heavy Industries Co., Ltd. His research interests include electric-powered transportation systems, PRT (Personal Rapid Transit), system engineering. 